\title{
Macrófitas aquáticas do Município de General Carneiro, Paraná, Brasil
}

\author{
Armando Carlos Cervit ${ }^{1,2}$, Cleusa Bona ${ }^{1,2,5}$, Maria Cecília de Chiara Moço ${ }^{4}$ \& Leonardo von Linsingen ${ }^{3}$ \\ ${ }^{1}$ Centro Politécnico, Setor de Ciências Biológicas, Departamento de Botânica, \\ Universidade Federal do Paraná - UFPR, \\ CP 19031, CEP 81531-990, Curitiba, PR, Brasil, www.bio.ufpr.br \\ ${ }^{2}$ Produtividade, Conselho Nacional de Desenvolvimento Científico e Tecnológico - CNPQ \\ ${ }^{3}$ Conservação da Natureza da Faculdade de Jaguariaíva, Setor de Ciências Agrárias, Engenharia Florestal \\ ${ }^{4}$ Universidade Federal do Rio Grande do Sul - UFRGS \\ ${ }^{5}$ Autor para correspondência: Cleusa Bona,e-mail: cleusabona@ufpr.br
}

CERVI, A.C., BONA, C., MOÇO, M.C.C. \& Von LINSINGEN, L. Aquatic macrophytes at General Carneiro, Parana State, Brazil. Biota Neotrop. 9(3): http://www.biotaneotropica.org.br/v9n3/en/abstract?inventory+bn0 0409032009.

\begin{abstract}
A floristic study of macrophyte communities was conducted at General Carneiro township, situated in

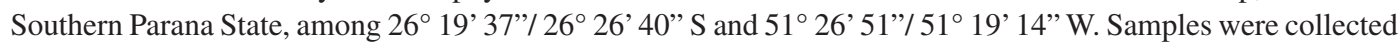
at São Pedro, São Pedro II, Santa Cândida and Lageado Grande farms, between the rivers São Pedro, São João, São Manoel, Iratim, Lajeado Grande and Neno, in order to study the flora of the rivers and ponds in this region. Macrophyte samples were randomly collected between 2004 and 2006 and registered in the UPCB and MBM herbaria. A total of 45 families was found, 42 of them being angiosperms, 70 genera and 117 species. The aquatic environments are floristicaly characterized by Cyperaceae, represented by 22 taxa, what means approximately $21 \%$ of all the encountered taxa, followed by Poaceae (8), Asteraceae (7) and Polygonaceae (6). Among the registered species we can highlight the first record in this state of Lilaeopsis minor Prez-Mor, Apiaceae.
\end{abstract}

Keywords: hydrophytes, aquatic plants, floristic survey, Cyperaceae, Poaceae.

CERVI, A.C., BONA, C., MOÇO, M.C.C. \& Von LINSINGEN, L. Macrófitas aquáticas do Município de General Carneiro, Paraná, Brasil. Biota Neotrop. 9(3): http://www.biotaneotropica.org.br/v9n3/pt/abstract?i nventory+bn00409032009.

Resumo: Foi realizado o estudo florístico das macrófitas do Município de General Carneiro, localizado no sul

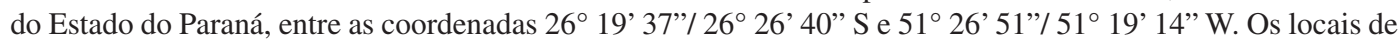
coleta foram as fazendas São Pedro, São Pedro II, Santa Cândida e Lageado Grande, entre os Rios São Pedro, São João, São Manoel, Iratim, Lajeado Grande e Neno. Com a finalidade de se conhecer a flora dos rios e lagoas da região foram realizadas coletas aleatórias das macrófitas entre 2004 e 2006. Os exemplares encontramse depositados no herbário UPCB e MBM. Registraram-se 45 famílias, 42 de angiospermas, 70 gêneros e 117 espécies. Os ambientes aquáticos são caracterizados floristicamente pelas Cyperaceae, representada por 22 táxons, o que significa cerca de $21 \%$ de todos os táxons encontrados, seguidos pelas Poaceae (8), Asteraceae (7) e Polygonaceae (6). Dentre as espécies registradas podemos destacar o primeiro registro para o estado de Lilaeopsis minor Prez-Mor., Apiaceae.

Palavras-chave: hidrófitas, plantas aquáticas, levantamento florístico, Cyperaceae, Poaceae. 


\section{Introdução}

O importante papel ecológico das macrófitas aquáticas como fonte de alimento, local de refúgio para diversas espécies de vertebrados e invertebrados e na ciclagem de nutrientes tem sido enfatizado em vários ecossistemas aquáticos continentais (Esteves 1998). Acreditase que estes organismos assumam papel ainda mais importante em ecossistemas rasos, como as planícies de inundação, onde colonizam extensas áreas e apresentam elevadas taxas de produção primária (Neiff 1986, 1978).

As macrófitas aquáticas são definidas por Cook (1996) como vegetais visíveis a olho desarmado, cujas partes fotossintetizantes ativas estão permanentemente ou por diversos meses, todos os anos, total ou parcialmente submersas em água doce, ou ainda flutuantes na mesma. Desempenham um importante papel nos ecossistemas aquáticos, cumprindo, juntamente com as microalgas, o papel de produtoras primárias, participando da ciclagem e estocagem de nutrientes, da formação de detritos orgânicos e do controle da poluição e da eutrofização artificial (Esteves \& Camargo 1986, Pott \& Pott 2000). Além disso, promovem a diversificação de habitats, criando locais de abrigo e alimentação para fauna diversificada e também como substrato natural para a formação de perifíton. Em rios e riachos, as macrófitas influenciam na sedimentação e retenção de nutrientes, nas características físicas e químicas da água, assim como, em alguns casos, afetar significativamente a velocidade de fluxo da água (Esteves 1998).

O Brasil possui a maior rede hidrográfica do mundo, sendo os ecossistemas aquáticos (fluviais, lacustres permanentes ou temporários) de grande representatividade dentre os ecossistemas brasileiros. A vegetação a eles associada compreende não apenas as hidrófitas propriamente ditas, como também as plantas que periodicamente estão submersas em diversos níveis ou as que margeiam estes ambientes, como por exemplo, as espécies anfíbias. Em regiões tropicais as macrófitas aquáticas são abundantes, graças às condições ambientais favoráveis ao seu crescimento (Pott et al. 1992).

Em nenhuma outra parte do mundo existem tantas espécies aquícolas como no Brasil (Hoehne 1955). No entanto, a bibliografia existente sobre o assunto não condiz com a grande diversidade de macrófitas (Bove et al. 2003). Thomaz \& Bini (2003) ressaltam a escassez de trabalhos realizados em rios brasileiros e o grande descaso sobre esses ecossistemas. Pompêo \& Moschini (2003) também enfatizaram que o número de publicações é reduzido em relação à grande área potencial de ocupação de macrófitas aquáticas no território nacional e reforçaram a necessidade da ampliação dos estudos com macrófitas aquáticas.

As áreas alagadas compreendem aproximadamente 6,5\% da superfície do território brasileiro (Esteves 1998) e representam um sistema ainda pouco conhecido e bastante ameaçado pela ação do homem. Segundo Tiner (1991), a ocorrência de espécies vegetais pode ser drasticamente modificada pela interferência humana. A distribuição e abundância de muitas plantas podem ser significativamente afetadas por práticas de silvicultura, atividades agrícolas, desenvolvimento urbano, projetos de drenagem, poluição e outras ações antrópicas.

Os estudos sobre as macrófitas brasileiras são insuficientes e estão concentradas em poucas áreas do país (Cordazzo \& Seelinger 1988, Notare 1992, Irgang \& Gastal 1996, Scremin-Dias et al. 1999, Pott \& Pott 2000, Pompêo \& Moschini-Carlos 2003, Thomaz \& Bini 2003). No Paraná ainda há uma vaga idéia das espécies de macrófitas aquáticas ocorrentes (Cervi et al. 1983).

O estudo dos ambientes aquáticos é fator relevante e imprescindível para possibilitar a sua preservação, assim como o seu manejo. São ecossistemas que possuem características muito particulares e qualquer alteração pode gerar respostas por vezes não desejadas, influenciando no desenvolvimento de espécies oportunistas, muitas vezes necessitando de manejo, sendo bons bioindicadores (Pompêo 2008).

Os ecossistemas aquáticos possuem grande representatividade na região de General Carneiro, porém nunca foi alvo de estudos. Este trabalho visa preencher essa lacuna, através da identificação dos táxons ali existentes e suas respectivas formas biológicas.

\section{Material e Métodos}

As coletas foram realizadas na região sul do Estado, Município de General Carneiro, entre as coordenadas $26^{\circ} 19^{\prime} 37^{\prime \prime}-26^{\circ} 26^{\prime} 40^{\prime \prime} \mathrm{S}$ e $51^{\circ} 26^{\prime} 51^{\prime \prime}-51^{\circ} 19^{\prime} 14^{\prime}$ ' W, altitude entre 1.000 e $1.150 \mathrm{~m}$, nas fazendas São Pedro, São Pedro II, Santa Cândida e Lageado Grande, totalizando 16 expedições botânicas. As coletas foram concentradas nos principais rios, brejos e lagoas que ocorrem na região, entre eles os Rios São Pedro, São João, São Manoel, Iratim, Lajeado Grande e Arroio Neno (Salto Amazonas) (Figura 1). Foram feitas coletas em cerca de oito lagoas (tamanho e profundidade variados), seis represas, sendo a maior a Represa das Araucárias, na sede da Fazenda Santa Cândida (comp. 1550 m, larg. 490 m, prof. 5,50 m, área: 75,95 ha), e a menor a Represa 15 (comp. 65 m, larg. 40 m, prof. $0,80 \mathrm{~m}$, área: $0,26 \mathrm{ha}$ ), três banhados e três lagoas temporárias (d. $40 \mathrm{~m}$, prof. $0,8 \mathrm{~m}$ ).

O material coletado foi herborizado segundo a metodologia tradicional utilizada para plantas terrestres (Mori et al. 1989) ou, quando delicadas, as plantas foram distendidas sobre papel sulfite em bandeja com água, cobertas com papel celofane, prensadas em jornal e secas em estufa. As exsicatas foram depositadas nos herbários UPCB e MBM. Foram coletadas, exclusivamente, plantas que se encontravam na água ou em solo úmido de ambientes lacustres e fluviais. Ganchos foram utilizados, quando necessário, para coletar exemplares de espécies submersas.

Foi dedicada especial atenção aos dados de coleta, que possuem grande importância nos vegetais hidrófilos, como profundidade, forma biológica (Irgang et al. 1984), coloração das partes vegetativas e reprodutivas e posição das flores e dos frutos em relação à lâmina d'água. Os ambientes distribuídos em lagoas temporárias, lagoas permanentes, represas, brejo permanente, brejo estacional e rio. Para o enquadramento sistemático/taxonômico para as famílias de angiospermas foi utilizado o proposto por APG II (2003).

\section{Resultados e Discussão}

Foram registradas 117 espécies, distribuídas em 74 gêneros e 45 famílias. Os ambientes aquáticos apresentam o predomínio de representantes da família Cyperaceae, com 22 táxons, o que significa $21 \%$ de todos os táxons encontrados. Outras famílias com maior número de representantes são: Poaceae (8), Asteraceae (7) e Polygonaceae (6). Referente às formas de vida a maioria das espécies são emergentes (57) (Tabela 1). Comparando-se com a nota prévia das Macrófitas aquáticas do Paraná publicado por Cervi et al. (1983) houve um aumento de 85 espécies, no entanto sabe-se que esse número é muito maior, visto que para o Rio Grande do Sul foram registradas entre 400 e 500 espécies de macrófitas aquáticas (Irgang \& Gastal 1996), e cerca de 247 para o Pantanal Sul-Matogrossense (Pott \& Pott 2000). Esses números evidenciam a necessidade extrema de se fazer um levantamento mais amplo das macrófitas aquáticas do estado do Paraná.

As famílias e gêneros predominantes em cada forma biológica são: 


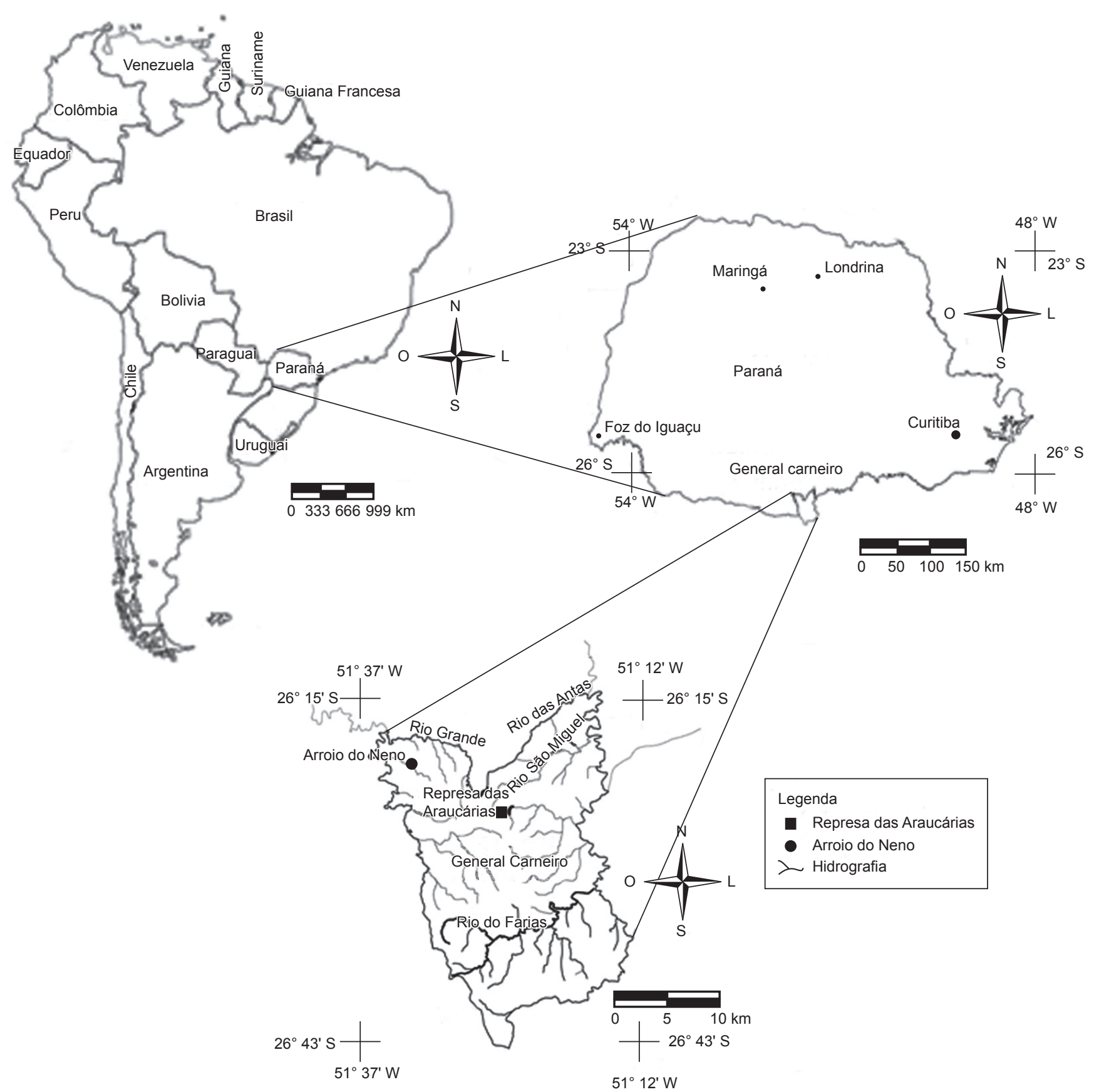

Figura 1. Localização do Município de General Carneiro - PR onde foram concentradas as coletas de macrófitas aquáticas. Mapa cedido por Pavan (2008). Figure 1. Location of General Carneiro township - Parana State, where the collects of aquatic macrophytes were concentrated. Map given by Pavan (2008).

Emergente (E) - Asteraceae, Lamiaceae, Rubiaceae, Poaceae com vários gêneros, Orchidaceae com o gênero Habenaria e Onagraceae com o gênero Ludwigia e Polygonaceae com o gênero Polygonum.

Anfíbia (A) - Campanulaceae, diversas Cyperaceae, Commelinaceae, Iridaceae, Juncaceae, Lamiaceae, Lythraceae, Melastomataceae (Tibouchina), uma Menianthaceae, algumas Poaceae, uma Urticaceae e Xyridaceae.

Flutuante fixa (FF) - Ranunculaceae, Nymphaeaceae, Araliaceae (Hidrocotyle).
Flutuante livre (FL) - Araceae.

Submersa fixa (SF) - Podostemaceae, Potamogetonaceae, Characeae, uma espécie de Pontederiaceae e uma de Ruppiaceae.

Submersa livre (SL) - Lentibulariaceae.

Dentre as espécies pode-se destacar o primeiro registro para o Estado de Lilaeopsis minor, da família Apiaceae. As espécies de ocorrência mais ampla foram Hydrocotyle ranunculoides, Nynphaea caerulea, Hypericum brasiliense, Eleocharis nana, Senecio jurgensii, Juncus microcephalus, Utricularia foliosa, Polygonum 
Tabela 1. Lista das espécies coletadas em General Carneiro e depositadas no Museu Botânico Municipal - Herbário MBM e no Departamento de Botânica da UFPR - Herbário UPCB, Curitiba, Paraná. 1. lagoa temporária; 2. lagoa permanente; 3. represa; 4. brejo permanente; 5. brejo estacional; 6. Rio. Coletor: $\mathrm{CB}=$ Cleusa Bona e $\mathrm{ACC}=$ Armando Carlos Cervi.

Table 1. List of species collected in General Carneiro and deposited at the Museu Botânico Municipal - MBM and at the Department of Botany of UFPR UPCB, Curitiba, Parana State. 1. temporary pond, 2. permanent pond, 3. dam, 4. Permanent marsh, 5. Seasonal marsh, 6. River. Plant collector: CB = Cleusa Bona e ACC = Armando Carlos Cervi.

\begin{tabular}{|c|c|c|}
\hline Família/espécie & Forma de vida/ambiente & $\mathbf{n}^{0}$ Col. \\
\hline \multicolumn{3}{|l|}{ ACANTHACEAE } \\
\hline Hygrophila costata Nees & Emergente/4 & CB 271 \\
\hline Hygrophila helodes Nees & Emergente/2, 4 & CB 235 \\
\hline \multicolumn{3}{|l|}{ ALISMATACEAE } \\
\hline Echinodorus grandiflorus (Cham. \& Schltdl.) Micheli & Anfíbia, emergente/5 & CB 164 \\
\hline \multicolumn{3}{|l|}{ ALSTROEMERIACEAE } \\
\hline Alstroemeria isabellana Herb. & Emergente/4 & CB 268 \\
\hline \multicolumn{3}{|l|}{ AMARANTHACEAE } \\
\hline Alternanthera reineckii Briq. & Anfíbia/3 & CB 246 \\
\hline \multicolumn{3}{|l|}{ APIACEAE } \\
\hline Lilaeopsis minor Prez-Mor. & Submersa fixa/3, 6 & ACC 8694 \\
\hline \multicolumn{3}{|l|}{ ARACEAE } \\
\hline Wollffiella oblonga (Phil.) Hegelm. & Flutuante livre/2 & ACC 8691 \\
\hline Wolffia brasiliensis Wedd. & Flutuante livre/2 & CB 375 \\
\hline Lemna minuta Kunth & Flutuante livre/6 & CB 142 \\
\hline \multicolumn{3}{|l|}{ ARALIACEAE } \\
\hline Hydrocotyle ranunculoides L.f. & Flutuante fixa/4, 6 & CB 243 \\
\hline Hydrocotyle verticillata Thumb. & Flutuante fixa/4, 6 & CB 172 \\
\hline \multicolumn{3}{|l|}{ ASTERACEAE } \\
\hline Barrosoa candolleana (Hook. \& Arn.) R.M.King \& H.Rob. & Emegente/1 & ACC 8856 \\
\hline Barrosoa betoniciiformis (DC.) R.M. King \& H. Rob. & Emergente/5 & CB 266 \\
\hline Erigeron tweediei Hook. \& Arn. & Emergente/5 & ACC 8887 \\
\hline Hypochoeris lutea (Vell.) Britton & Emergente/1 & CB 292 \\
\hline Jaegeria hirta (Lag.) Less. & Emergente/6 & CB 220 \\
\hline Lessingianthus glabratus (Less.) H. Rob. & Emergente/5 & CB 265 \\
\hline Senecio juergensii Mattf. & Emergente/4, 6 & ACC 8671 \\
\hline \multicolumn{3}{|l|}{ BEGONIACEAE } \\
\hline Begonia setosa Klotzsch & Emergente/5 & ACC 8839 \\
\hline \multicolumn{3}{|l|}{ BRASSICACEAE } \\
\hline Cardamine bonariensis Pers. & Emergente/6 & CB 222 \\
\hline \multicolumn{3}{|l|}{ CAMPANULACEAE } \\
\hline Lobelia hassleri A. Zahlbr. & Anfíbia/5 & ACC 8838 \\
\hline Lobelia hederacea (Cham.) G. Don & Anfíbia/5 & CB 251 \\
\hline Siphocampylus verticillatus (Cham.) G. Don & Anfíbia/5, 6 & CB 302 \\
\hline \multicolumn{3}{|l|}{ COMMELINACEAE } \\
\hline Commelina diffusa Burm. f. & Anfíbia/3 & CB 244 \\
\hline Floscopa glabrata (Kunth) Hassk. & Anfíbia/2 & CB 258 \\
\hline Tradescantia fluminensis Vell. & Anfíbia/2 & ACC 8680 \\
\hline \multicolumn{3}{|l|}{ CYPERACEAE } \\
\hline Carex brasiliensis A. St.-Hil. & Anfíbia/3, 6 & CB 190 \\
\hline Carex bonariensis Desf. ex Poir. & Anfíbia/2 & ACC 8676 \\
\hline Carex purpureovaginata Boeck. & Anfíbia, emergente/3 & CB 186 \\
\hline Cyperus consanguineus Kunth & Anfíbia/4 & ACC 8651 \\
\hline Eleocharis dunensis Kük. & Emergente/4 & CB 156 \\
\hline Eleocharis flavescens (Poir.) Urb. & Emergente/4 & CB 360 \\
\hline Eleocharis maculosa (Vahl) Roem. \& Schult. & Emergente/2 & CB 340 \\
\hline
\end{tabular}


Tabela 1. Continuação...

\begin{tabular}{|c|c|c|}
\hline Família/espécie & Forma de vida/ambiente & $\mathbf{n}^{0}$ Col. \\
\hline Eleocharis montana (Vahl) Roem. \& Schult. & Emergente/3 & CB 361 \\
\hline Eleocharis minarum Boeck & Emergente/1 & CB 157 \\
\hline Eleocharis nana Kunth & Submersa fixa/1 & CB 288 \\
\hline Eleocharis niederleinii Boeck & Anfíbia/1 & CB 371 \\
\hline Eleocharis kleinii Barros & Anfíbia/5 & CB 369 \\
\hline Eleocharis obtusetrigona (Lindl. \& Nees) Steud. & Emergente/3 & CB 162,368 \\
\hline Eleocharis rabenii Boeck. & Anfíbia/5 & ACC 8867 \\
\hline Eleocharis radicans (Poir) Kunth & Emergente/5 & CB 263 \\
\hline Eleocharis sellowiana Kunth & Anfíbia/1 & CB 370 \\
\hline Eleocharis subarticulata (Nees) Boeck. & Anfíbia/5 & CB 362 \\
\hline Eleocharis viridans Kük. ex Osten & Emergente/5 & ACC 8657 \\
\hline Kyllinga odorata Vahl. & Anfíbia/5 & CB 332 \\
\hline Pycreus lanceolatus Poir. C.B. Clarke & Emergente/2 & CB 354 \\
\hline Rhynchospora corymbosa (Ness.) Kunth & Emergente/4 & ACC 8675 \\
\hline Rynchospora gollnieri Boeck. & Emergente/2 & CB 311 \\
\hline \multicolumn{3}{|l|}{ ERIOCAULACEAE } \\
\hline Eriocaulon ligulatum L.B. Sm. & Emergente/4 & CB 306 \\
\hline Paepalanthus caldenisis Silveira & Emergente/6 & CB 187 \\
\hline Syngonanthus caulescens (Poir.) Ruhland & Emergente/5 & CB 264 \\
\hline \multicolumn{3}{|l|}{ EUPHORBIACEAE } \\
\hline Croton pallidus Müll. Arg. & Emergente/4 & CB 306 \\
\hline \multicolumn{3}{|l|}{ HALORAGACEAE } \\
\hline Myriophillum aquaticum (Vell.) Verdc. & Subm. fixa, emergente/3 & CB 201 \\
\hline \multicolumn{3}{|l|}{ HYPERICACEAE } \\
\hline Hypericum brasiliense Choisy & Anfíbia/1, 4 & ACC 8670,8851 \\
\hline \multicolumn{3}{|l|}{ IRIDACEAE } \\
\hline Sisyrinchium palmifolium $\mathrm{L}$. & Anfíbia/4 & CB 206, 330 \\
\hline Sisyrinchium rombonis Fost. & Anfíbia/1, 2 & ACC 8884 \\
\hline Sisyrinchium restioides Spreng. & Anfíbia/1 & CB 337 \\
\hline Sisyrinchium vaginatum Spreng. & Emergente/1 & CB 327 \\
\hline \multicolumn{3}{|l|}{ JUNCACEAE } \\
\hline Juncus effusus L. & Anfíbia/4, 5 & CB 171 \\
\hline Juncus microcephalus Kunth & Anfíbia/5, 1 & CB 160,175 \\
\hline \multicolumn{3}{|l|}{ LAMIACEAE } \\
\hline Hyptis lappulacea Mart. ex Benth. & Anfíbia/6 & ACC 8871 \\
\hline Hyptis uliginosa A.St.-Hil. ex Benth. & Anfíbia/2 & CB 260 \\
\hline Scutellaria racemosa Pers & Anfíbia/2 & CB 208 \\
\hline \multicolumn{3}{|l|}{ LENTIBULARIACEAE } \\
\hline Utricularia foliosa $\mathrm{L}$. & Submersa livre/2, 4 & CB 161 \\
\hline Utricularia gibba $\mathrm{L}$. & Submersa livre/2, 5 & CB 225 \\
\hline \multicolumn{3}{|l|}{ LINDERNIACEAE } \\
\hline Micranthemum umbrosum S.F. Blake & Emergente/3 & CB 236 \\
\hline \multicolumn{3}{|l|}{ LYTHRACEAE } \\
\hline Cuphea carthagenensis (Jacq.) J.F. Macbr. & Anfíbia/5,2 & CB 245 \\
\hline Cuphea glutinosa Cham. \& Schltdl. & Anfíbia/6,2 & CB 191 \\
\hline \multicolumn{3}{|l|}{ MAYACACEAE } \\
\hline Mayaca sellowiana Kunth & Anfíbia/2 & ACC 8845 \\
\hline \multicolumn{3}{|l|}{ MELASTOMATACEAE } \\
\hline Tibouchina cerastifolia (Naudin) Cogn. & Anfíbia/2 & CB 249 \\
\hline Tibouchina clinopodifolia (DC.) Cogn. & Anfíbia/2 & CB 255 \\
\hline
\end{tabular}


Tabela 1. Continuação...

\begin{tabular}{|c|c|c|}
\hline Família/espécie & Forma de vida/ambiente & $\mathbf{n}^{0}$ Col. \\
\hline Tibouchina herbacea (DC.) Cogn. & Anfíbia/2 & CB 8854 \\
\hline Tibouchina ursina (Cham.) Cogn. & Anfíbia/5 & CB 275 \\
\hline \multicolumn{3}{|l|}{ MENIANTHACEAE } \\
\hline Nymphoides indica (L.) Kuntze & Anfíbia/1, 2 & CB 283, 295 \\
\hline \multicolumn{3}{|l|}{ MYRCINACEAE } \\
\hline Anagallis filiformis Cham. \& Schltdl. & Emergente/2 & CB 280 \\
\hline Anagallis minimus L. & Emergente/6 & CB 194 \\
\hline \multicolumn{3}{|l|}{ NYMPHAEACEAE } \\
\hline Nymphaea caerulea Savigny & Flutuante fixa/2, 3 & CB 141 \\
\hline \multicolumn{3}{|l|}{ ONAGRACEAE } \\
\hline Ludwigia leptocarpa (Nutt.) H. Hara & Emergente/2, 6 & CB 241 \\
\hline Ludwigia longifolia (DC.) H. Hara & Emergente/2 & CB 300 \\
\hline Ludwigia peruviana (L.) Hover & Emergente/2 & CB 232 \\
\hline Ludwigia sericea (Camb.) H. Hara & Emergente/6 & CB 334 \\
\hline Ludwigia uruguayensis (Camb.) H. Hara & Emergente/2 & CB 198 \\
\hline \multicolumn{3}{|l|}{ ORCHIDACEAE } \\
\hline Cyclopogon apricus (Lindl.) Schltr. & Anfíbia/5 & ACC 8656 \\
\hline Habenaria macronectar (Vell.) Hoehne & Emergente/6 & CB 303 \\
\hline Habenaria montevidensis Spreng. & Emergente/6 & ACC 8868 \\
\hline Habenaria parviflora Lindl. & Emergente/2 & CB 250,259 \\
\hline \multicolumn{3}{|l|}{ OROBANCHACEAE } \\
\hline Agalinis tenella Pennell & Submersa fixa/1 & CB 280 \\
\hline \multicolumn{3}{|l|}{ PLANTAGINACEAE } \\
\hline Gratiola peruviana $\mathrm{L}$. & Anfíbia/4, 6 & ACC 8841 \\
\hline Callitriche stagnalis Scop. & Flutuante fixa/1 & CB 204, 322 \\
\hline \multicolumn{3}{|l|}{ POACEAE } \\
\hline Agrostis ramboi Parodi & Emergente/6 & CB 218 \\
\hline Andropogon lateralis Nees. & Emergente/4 & CB 299 \\
\hline Cynodon dactylon (L.) Pers. & Anfíbia/3 & CB 323 \\
\hline Luziola peruviana Juss. ex J.F. Gurel & Emergente/2 & CB 223, 347 \\
\hline Panicum helobium Wez ex Henrad & Emergente/4, 6 & CB 215,244 \\
\hline Panicum villosum $\mathrm{Sw}$. & Emergente/6 & CB 233 \\
\hline Pseudechinolaena polystachya (Kunth) Stapf & Anfíbia/5 & CB 279 \\
\hline Sacciolepis vilvoides (Trin.) Chase & Anfíbia/5 & CB 168 \\
\hline \multicolumn{3}{|l|}{ PODOSTEMACEAE } \\
\hline Podostemum distichum (Cham.) Wedd. & Submersa fixa/6 & ACC 8877 \\
\hline \multicolumn{3}{|l|}{ POLYGONACEAE } \\
\hline Polygonum acuminatum Kunth & Anfíbia/3 & CB 277 \\
\hline Polygonum hydropiperoides Michx. & Emergente/2 & CB 173 \\
\hline Polygonum meisnerianum Cham. \& Schltdl. & Emergente/2 & CB 165 \\
\hline Polygonum persicaria $\mathrm{L}$. & Emergente/2 & CB 276 \\
\hline Polygonum punctatum Elliot & Emergente/2 & CB 199 \\
\hline Polygonum rubricaule Cham. & Emergente/6 & CB 213 \\
\hline \multicolumn{3}{|l|}{ PONTEDERIACEAE } \\
\hline Heteranthera reniformis Ruiz \& Pavon. & Emergente/2, 6 & CB 219 \\
\hline Heteranthera zosterifolia Mart. & Submersa fixa/2, 6 & CB 324 \\
\hline Pontederia lanceolata $\mathrm{Nutt}$ & Emergente/2 & CB 309 \\
\hline \multicolumn{3}{|l|}{ POTAMOGETONACEAE } \\
\hline Potamogeton montevidensis A. Bennett & Submersa fixa/6 & ACC 8696 \\
\hline Potamogeton polygonus Cham. \& Schltdl. & Submersa fixa/2, 3,6 & CB 151,202 \\
\hline
\end{tabular}


Tabela 1. Continuação...

\begin{tabular}{|c|c|c|}
\hline Família/espécie & Forma de vida/ambiente & $\mathbf{n}^{0}$ Col. \\
\hline \multicolumn{3}{|l|}{ RANUNCULACEAE } \\
\hline Ranunculus flagelliformis Nakai & Flutuante fixa/1 & CB 328, 284 \\
\hline \multicolumn{3}{|l|}{ RUBIACEAE } \\
\hline Galianthe cymosa (Cham.) E.L. Cabral \& Bacigalupo & Anfíbia, emergente/4 & ACC 8862 \\
\hline Galianthe dichasia (Sucre \& Costa) E.L. Cabral & Anfíbia, emergente/6 & CB 148 \\
\hline Oldenlandia thesiifolia (A.St.-Hil.) Schum. & Emergente/2 & CB 348 \\
\hline \multicolumn{3}{|l|}{ RUPPIACEAE } \\
\hline Ruppia cirrhosa (Petagna) Grande & Submersa fixa/2, 3, 6 & CB 315,318 \\
\hline \multicolumn{3}{|l|}{ URTICACEAE } \\
\hline Boehmeria cylindrica (L.) Sw. & Anfíbia/4 & ACC 8861 \\
\hline \multicolumn{3}{|l|}{ XYRIDACEAE } \\
\hline Xyris jupicai L.C. Rich. & Anfíbia/2 & CB 248 \\
\hline Xyris stenophylla L.A. Nilsson & Anfibia/4 & CB 310 \\
\hline \multicolumn{3}{|l|}{ SALVINIACEAE } \\
\hline Salvinia auriculata Aubl. & Flutuante livre/2 & CB 205 \\
\hline \multicolumn{3}{|l|}{ SPHAGNACEAE } \\
\hline Sphagnum recurvum $\mathrm{P}$. Beauv. & Anfíbia/5 & CB 144 \\
\hline \multicolumn{3}{|l|}{ CHARACEAE } \\
\hline Chara martiana A. Braun ex Wallman & Submersa fixa $/ 3$ & CB 352 \\
\hline Nitella furcata C. Agardh & Submersa fixa/3 & CB 237 \\
\hline Nitella translucida (Pears) C. Agardh & Submersa fixa/3 & CB 184 \\
\hline
\end{tabular}

meissnerianum, Polygonum hydropireoides e Potamogeton polygonus. Outras espécies apresentaram ocorrência restrita de ambiente lótico como Podostemum distichum e Potamogeton montevidensis ou de ambiente lêntico como Nymphoides indica e Nymphaea caerulea.

A flora estudada é heterogênea e apresenta 26 espécies ruderais ou oportunistas, de acordo com Lorenzi (2000), isso é facilmente explicado pela grande produção de sementes, alta capacidade de adaptação e resistência das mesmas. Espécies comuns em ambientes com má qualidade de água - Salvinia auriculata, Ecchinodorus grandiflorus e Polygonum spp. - (Pedralli 2003) também foram registradas.

Fatores como a heterogeneidade ambiental, as oscilações do nível da água, a velocidade da correnteza e a quantidade de nutrientes disponíveis podem afetar significativamente a riqueza de macrófitas aquáticas nos corpos de água (Thomas \& Bini 2003). Além disso, as listagens podem ainda ser afetadas pela quantidade de áreas e períodos amostrados, além das intervenções antrópicas nos rios e lagos (Rorslett 1991).

Pompêu \& Moschini-Carlos (2003) evidenciaram que a massa de macrófitas aquáticas submersas é baixa quando comparada com a de macrófitas aquáticas emersas em ambientes lênticos do interior de São Paulo. Para as lagoas de General Carneiro esse padrão também ocorre. Apenas algumas macrófitas como Potamogeton polygonus e as espécies de Characeae encontram-se em profundidades maiores, formando agrupamentos homogêneos e característicos destas regiões da lagoa. As políticas de conservação têm sido baseadas largamente somente na riqueza de espécies. Sugere-se que a variabilidade ambiental e a substituição de espécies também sejam levadas em consideração no delineamento destas políticas (Tockner 1999, Ward et al. 1999) e estes componentes da diversidade total devem ser incorporados nas investigações de ambientes aquáticos.

\section{Conclusão}

O levantamento florístico das macrófitas de General Carneiro proporcionou gerar uma lista de espécies, fator indispensável na conservação de táxons pouco conhecidos. O número de espécies coletadas define um ambiente conservado, porém esforços relacionados à conservação não foram verificados e caso não seja empregado nenhuma medida as espécies podem estar ameaçadas principalmente pela invasão de espécies exóticas como o Pinus nas margens dos rios e lagos. Após esse levantamento ficou evidente a necessidade da continuação do levantamento das macrófitas aquáticas para o estado do Paraná, pois o número de espécies está aquém do esperado.

\section{Agradecimentos}

Agradecemos ao CNPq (Conselho Nacional de Desenvolvimento Científico e Tecnológico) pelo apoio financeiro e ao Instituto Ecoplan e Fundação Pizzatto pelo apoio logístico durante as coletas.

\section{Referências Bibliográficas}

Angiosperm Phylogeny Group - APG. 2003. An update of the Angiosperm Phylogeny Group classification for the orders and families of flowering plants: APG II. Bot. J. Linnean Soc. 141(141):399-436.

BOVE, C.P., BRAGANÇA-GIL, A.S., MOREIRA, C.B. \& ANJOS, R.F.B. 2003. Hidrófitas fanerogâmicas de ecossistemas aquáticos temporários da planície costeira do Estado do Rio de Janeiro, Brasil. Acta Bot. Bras. 17(1):119-135.

CERVI, A.C., HATSCHBACH, G. \& GUIMARÃES, O.A. 1983. Nota prévia sobre plantas aquáticas (fanerogâmicas) do Estado do Paraná (Brasil). Bol. Mus. Bot. Mun. 58:1-17.

COOK, C.D.K. 1996. Aquatic and wetland plants of India. Oxford University Press, Oxford.

CORDAZZO, C.V. \& SEELIGER, U. 1988. Guia ilustrado da vegetação costeira no extremo sul do Brasil. Furg, Rio Grande.

ESTEVES, F.A. 1998. Fundamentos de limnologia. Interciência/FINEP, Rio de Janeiro. 
ESTEVES, F.A. \& CAMARGO, A.F.M. 1986. Sobre o papel das macrófitas aquáticas na estocagem e ciclagem de nutrientes. Acta Limnol. Bras. 1(1):273-298

HOEHNE, F.C. 1955. Plantas aquáticas. Secretaria de Agricultura de São Paulo, São Paulo.

IRGANG, B.E., PEDRALLI, G. \& WAECHTER, J.L. 1984. Macrófitas aquáticas da estação ecológica do Taim, Rio Grande do Sul, Brasil. Roessléria. 6(1):395-404.

IRGANG, B.E. \& GASTAL Jr., C.V.S. 1996. Plantas aquáticas da planície costeira do Rio Grande do Sul. Edição dos autores, Porto Alegre.

LORENZI, H. 2000. Plantas Daninhas do Brasil: Terrestres, aquáticas e tóxicas. 3 ed. Instituto Plantarum, Nova Odessa.

MORI, S.A., SILVA, L.A.M., LISBOA, G. \& CORADIN, L. 1989. Manual de manejo do herbário fanerogâmico. CEPAAC, Ilhéus.

NEIFF, J.J. 1978. Fluctuaciones de la vegetacion acuatica en ambientes del valle de inundacion del Paraná medio. Physis. 38(95):41-53.

NEIFF, J.J. 1986. Aquatic plants of the Paraná River system. In The ecology of river systems (B.R. Davies \& K.F. Walker, eds.). Dr. W. Junk Publishers, Dordrecht, p. 557-571.

NOTARE, M. 1992. Plantas hidrófilas e seu cultivo em aquários. Sulamérica, Rio de Janeiro.

PAVAN, G. 2008. Diatomáceas perifíticas em Potamogeton montevidensis Arth. Bennett (Potamogetonaceae) em ambiente lótico e lêntico. Dissertação de Mestrado, Universidade Federal do Paraná, Paraná, p. 19.

PEDRALLI, G. 2003. Macrófitas aquáticas como bioindicadoras da qualidade da água: alternativa para usos múltiplos de reservatórios. In Ecologia e manejo de macrófitas aquáticas (S.M. Thomaz \& L.M. Bini, eds.). Universidade Estadual de Maringá, Maringá.
POMPÊO, M.L.M. \& MOSCHINI-CARLOS, V. 2003. Macrófitas aquáticas e perifíton: aspectos ecológicos e metodológicos. Rima, São Carlos.

POMPEO, M. 2008. Monitoramento e manejo de macrófitas aquáticas. Ecol. Bras. 12(3):406-424.

POTT, V.J., BUENO, N.C. \& SILVA, M.P. 1992. Levantamento florístico e fitossociológico de macrófitas aquáticas em lagoas da Fazenda Leque, Pantanal, MS. In Anais do VIII Congresso da Sociedade Botânica de São Paulo. Campinas: SBSP, p. 91-99.

POTT, V.J. \& POTT, A. 2000. Plantas aquáticas do Pantanal. Embrapa, Brasília.

RØRSLETT, B. 1991. Principal determinants of aquatic macrophyte richness in northern European lakes. Aquat. Bot. 39(1-2):173-193.

SCREMIN-DIAS, E., POTT, V.J., HORA, R.C. \& SOUZA, P.R. 1999. Nos jardins submersos da Bodoquena. UFMS, Campo Grande.

THOMAZ, S.M. \& BINI, L.M. 2003. Ecologia e manejo de macrófitas aquáticas. Universidade Estadual de Maringá, Maringá.

TINER, R.W. 1991. The concept of a hydrophyte for wetland identification. Bioscience. 41(4):236-247.

TOCKNER, K. 1999. The Danube restoration project: species diversity patterns across connectivity gradients in the floodplain system. Regul. Rivers Res. Manag. Chichester. 15(1-3):245-258.

WARD, J.M.J., STROMBERG, E.L., NOWELL, D.C. \& NUTTER Jr., F.W. 1999. Gray leaf spot: a disease of global importance in maize production. Plant Dis. 83(10):884-895.

Recebido em 09/09/08 Versão reformulada recebida em 25/06/09

Publicado em 01/07/09 